\title{
Vestibular-Balance Rehabilitation in Patients with Whiplash-Associated Disorders
}

Mohsen Ahadi* Zahra Naser Jamileh Abolghasemi

\begin{abstract}
Background and objective: Whiplash associated syndrome is one of the neck disorders that is accompanied by several chronic symptoms. Balancing problems arising from common disorders and dizziness are considered as manifestations that are time-consuming to evaluate and treat. The present study aims to investigate the effect of vestibular-balance rehabilitation using a test that can be used for differential diagnosis of these lesions by comparing dizziness handicap inventory (DHI) and Smooth Pursuit Neck Torsion (SPNT) scores.

Method: This was an analytical cross-sectional study with a two-stage design. Forty patients with whiplash-associated disorders were randomly divided into control and intervention groups. SPNT test and DHI evaluation were performed for both groups. For twenty people selected randomly in the intervention group, vestibular-balance rehabilitation was performed during 12 sessions. Finally, the SPNT and DHI tests were performed again to examine and compare the results.

Results: The results of this study showed that there was a statistically significant difference between the scores of smooth pursuit neck torsion gain in the SPNT test, the total score of DHI, the functional components of this questionnaire between the control and intervention groups after the implementation of the rehabilitation, and the differences in the physical, functional, and emotional components of the questionnaires of the control and intervention groups after rehabilitation exercises.
\end{abstract}

Conclusion: Vestibular-balance rehabilitation exercises can be an effective treatment for dizziness and improving the quality of life of a person suffering from whiplash-associated disorder, followed by the reduction in dizziness caused by disability.

Keywords: Vestibular balance; rehabilitation exercises; dizziness; whiplashs. 


\section{INTRODUCTION}

The whiplash syndrome is a complex disorder that is associated with the injury to the neck or its soft tissues resulting from sudden and forward acceleration due to the effect of hyperextension or hyper flexion of the neck. Several chronic symptoms such as tinnitus, neck ache, Temporo Mandibular Joint (TMJ) disorder, ataxia, neck stiffness, decrease in neck motion range, headache, concentration problems, psychosocial problems, and dizziness. The sum of these symptoms is known as Whiplash-Associated Disorder (WAD) ${ }^{1-4}$. Since there is no specific test for the differential diagnosis of this lesion among other cervical disorders, the Smooth Pursuit Neck Torsion (SPNT) test has recently been considered. Advantages of this test include its high sensitivity and specificity, lack of pain-pressure and other discomforts for the patient, and easy and convenient implementation using the oculomotor equipment ${ }^{4}$. Abnormal results in this test indicate abnormal neck proprioceptive inputs to the vestibular nuclei ${ }^{5}$. The basis of the SPNT test is the evaluation of neck proprioceptive inputs, Cervical Ocular Reflex (CCR), and Cervical Dizziness Collic Reflex (CDCR) ${ }^{6}$. By correct and timely diagnosis of interdisciplinary disorders through choosing the proper test and subsequent appropriate treatments and interventions, it is possible to affect the patient's quality of life significantly. Subjective dizziness with functional disorder affects the balance of most patients with this lesion. This disorder is hard to evaluate and treat clinically? Among the several treatments presented, the efficacy and positive impacts vestibular-balance rehabilitation have been proven ${ }^{8}$. The aim of rehabilitation is to re-establish symmetry and proprioceptive normal feedbacks from splenius cervicis muscle to the central nervous system? Despite the few and limited studies that have been done on patients with whiplash-associated disorder and therapeutic methods in these patients, the purpose of this study was to evaluate the efficacy and effectiveness of vestibular-balance rehabilitation in treating patients with WAD, and to implement the SPNT test as one of the methods for differential diagnosis of this disorder ${ }^{10}$.

\section{MATERIALS AND METHODS}

This research was approved by the Vice-Chancellor of Ethics Committee of Iran University of Medical Sciences (Code of Ethics: IR.IUMS.FMD.REC 1396.9413301003) as an applied study in analytical cross-sectional form, and was carried out using two stages by comparing the score of smooth pursuit neck torsion gain and total score and the score of components of dizziness handicap inventory before and after the vestibular-balance rehabilitation exercises. The subjects of this study were selected from the patients referred and hospitalized in the orthopedic and rheumatologic departments of Amir Alam and Hazrat Rasool Hospitals, Tehran, Iran during 2017-18. Forty patients with WAD including 15 women and 25 men with age group of 15-64 years old were randomly divided into control and intervention groups. At first, in order to randomization, numbers from 1 to 40 were randomly selected by software on the internet and were then divided into two groups. The list was then provided to the physician and the patients entered the study in turn. In the first stage, all patients in both control and intervention groups were subjected to the SPNT test in order to determine the Smooth Pursuit (SP) gain, SPNT gain, and the difference between these two gains (SPNT diff), and then completed $\mathrm{DHI}$ in order to evaluate total score and the components of this questionnaire. The implementation of the SPNT test was such that the patient was once in a normal situation and using the VNG device, the SP gain was calculated. In the next step, the patient's neck was in the 45-degree rotated position to the right and left, and the mean SPNT gain in both directions was calculated. In order to eliminate the involvement of the vestibular space between the rotation situations to the right and left, a short pause was given. Finally, the differences between the SPNT and SP (SPNT diff) were calculated. In cases where 45 degrees of neck rotation were causing pain, an angle of at least 30 degrees was used. DHI was used to assess dizziness, which consisted of functional, physical and emotional components. The questionnaire consisted of 25 items with three choices and each item had the following choices: yes (4 scores), sometimes (2 scores) and no (zero scores). For 20 patients in the intervention group, vestibular-balance rehabilitation exercises were performed during 12 50-minute sessions, twice a week for 6 weeks. Exercises included: standing on a foam surface and turning the head to the sides, walking on an inclined plane and turning the head to the sides, standing on a trampoline and moving the eyes to the sides, heel-to-toe walk on the line up to 5 meters, and standing on one leg. In more difficult levels, these exercises were done with closed eyes. In the second stage, after performing these exercises, SPNT test was performed again for both control and intervention groups to determine SP, SPNT, and SPNT diff. The DHI was then completed by the patients. Inclusion criteria were presence of vertigo in WAD patients with grades I, II or grade III based on Quebec Task Force Classification (QTFC), and the patients consent on participation in the study. The exclusion criteria were positive Hallpike test and patients non-willingness to participation and withdrawn from the study. The paired $t$ test and standard Chi-square tests were performed to establish the significance of the difference between the various above measurements in each group.

\section{RESULTS}

In this study, 40 patients with whiplash-associated disorder in the age group of 15-64 years were randomly divided into two groups: intervention $(n=20)$, and control $(n=20)$. There was no significant difference between the demographic and clinical data of the two groups (Table 1). In the first stage and before performing vestibularbalance rehabilitation exercises, there was no significant 
Table 1. Patients' demographic and clinical data.

\begin{tabular}{|c|c|c|c|c|}
\hline \multicolumn{2}{|c|}{ Variable } & \multirow{2}{*}{$\begin{array}{c}\text { Intervention } \\
6(30 \%)\end{array}$} & \multirow{2}{*}{$\begin{array}{l}\text { Control } \\
9(45 \%)\end{array}$} & \multirow{3}{*}{$\begin{array}{c}\text { P-Value } \\
0.074\end{array}$} \\
\hline Gendor & Female & & & \\
\hline Genaer & male & 14 (70\%) & $11(55 \%)$ & \\
\hline \multicolumn{2}{|c|}{ Age (year) } & $42.30 \pm 2.746$ & $44.75 \pm 2.747$ & 0.532 \\
\hline \multicolumn{2}{|c|}{ Duration of involvement (month) } & $31.65 \pm 5.613$ & $34.05 \pm 5.034$ & 0.752 \\
\hline
\end{tabular}

difference among the mean scores of the SP gain in normal condition, SPNT gain, and SPNT diff in both groups $(p>0.05)$. After performing vestibular-balance rehabilitation exercises, there was no significant difference between the mean score of SP in both groups. However, two SPNT and SPNT diff scores were significantly different in the control and intervention groups ${ }^{11}$. Also, the results of the first and second stages in the control group did not show a significant difference, while there was a significant difference between the mean of the results of the first and second stages in the intervention group (Table 2).In the first stage, there was no significant difference between the mean score of the components and the total score of the $\mathrm{DHI}$ in both groups $(p>0.05)$. After performing vestibularbalance rehabilitation exercises in intervention group, significant was observed difference between the mean total score and the score of functional, physical, and emotional components before and after the exercises. These values were not significantly different in the control group (Table 3).

\section{DISCUSSION}

Combining vestibular rehabilitation and balancing rehabilitation can be helpful in treating dizziness caused by WAD. Vestibular rehabilitation therapy exercises are aimed at reducing dizziness symptoms, adjusting proprioceptive afferents of the neck, and ocular exercises to improve the function of $\mathrm{VOR}^{12-14}$. Assessing the symptoms and the severity of dizziness by questionnaire can give us an accurate insight into the impact of treatment on the physical, functional and emotional problems of the patient. The results of a study by Tjell et al. ${ }^{6}$ reported the sensitivity and specificity of the SPNT test in WAD patients with dizziness $90 \%$ and $91 \%$, respectively, and recommended this test for diagnosis of WAD patients with dizziness symptoms. The aim of this study was to investigate the effect of vestibular-balance rehabilitation in patients with whiplash-associated disorder, using Dizziness Handicap Inventory (DHI) and Smooth Pursuit Neck Torsion (SPNT) test. 40 patients were studied in two groups including

Table 2. Comparison the results of the first and second stages in the two groups

\begin{tabular}{|c|c|c|c|c|}
\hline \multicolumn{2}{|c|}{ Variable } & Before & After & P-Value \\
\hline \multirow{2}{*}{ SP } & Intervention & $0.84 \pm 0.010$ & $0.85 \pm 0.009$ & $0.012^{\star}$ \\
\hline & Control & $0.82 \pm 0.013$ & $0.82 \pm 0.012$ & 0.92 \\
\hline \multicolumn{2}{|c|}{ P-Value } & 0.432 & 0.098 & - \\
\hline \multirow{2}{*}{ SPNT } & Intervention & $0.66 \pm 0.013$ & $0.77 \pm 0.011$ & $<0.0001^{*}$ \\
\hline & Control & $0.66 \pm 0.017$ & $0.66 \pm 0.017$ & 1 \\
\hline \multicolumn{2}{|c|}{ P-Value } & 0.757 & $<0.0001^{*}$ & - \\
\hline \multirow{2}{*}{ SPNT diff } & Intervention & $0.17 \pm 0.011$ & $0.08 \pm 0.007$ & $<0.0001^{*}$ \\
\hline & Control & $0.16 \pm 0.012$ & $0.16 \pm 0.013$ & 0.92 \\
\hline \multicolumn{2}{|c|}{ P-Value } & 0.683 & $<0.0001^{*}$ & - \\
\hline${ }^{*} \mathrm{P}<0.05$ is considere & evel & & & \\
\hline
\end{tabular}

Table 3. Comparison of mean total score and components of $\mathrm{DHI}$ in the two group0073

\begin{tabular}{|c|c|c|c|c|}
\hline \multicolumn{2}{|c|}{ Component } & Before & After & P-Value \\
\hline \multirow{2}{*}{ Physical } & Intervention & $16.80 \pm 1.755$ & $10.70 \pm 1.712$ & $<0.0001^{*}$ \\
\hline & Control & $14.20 \pm 1.457$ & $14.30 \pm 1.445$ & 0.95 \\
\hline \multicolumn{2}{|c|}{ P-Value } & 0.262 & 0.0116 & - \\
\hline \multirow{2}{*}{ Functional } & Intervention & $21.20 \pm 1.496$ & $10.70 \pm 1.267$ & $<0.0001^{*}$ \\
\hline & Control & $20.20 \pm 1.329$ & $19.90 \pm 1.277$ & 0.63 \\
\hline \multicolumn{2}{|c|}{ P-Value } & 0.620 & $<0.0001^{*}$ & - \\
\hline \multirow{2}{*}{ Emotional } & Intervention & $12.80 \pm 1.808$ & $8.20 \pm 1.428$ & $<0.0001^{*}$ \\
\hline & Control & $12.70 \pm 1.408$ & $12.70 \pm 1.423$ & 0.98 \\
\hline \multicolumn{2}{|c|}{ P-Value } & 0.92 & $<0.0001^{*}$ & - \\
\hline \multirow{3}{*}{ Total } & Intervention & $43.10 \pm 2.10$ & $29.60 \pm 2.855$ & $<0.0001^{*}$ \\
\hline & Control & $50.80 \pm 3.57$ & $46.90 \pm 1.965$ & 0.055 \\
\hline & P-Value & 0.071 & $<0.0001^{*}$ & - \\
\hline
\end{tabular}


intervention and control. Before performing vestibularbalance rehabilitation exercises, there was no significant difference among the mean scores of SP, SPNT, and SPNT diff in both groups $(p>0 / 05)$. After performing the exercises, there was a significant difference between the SPNT and SPNT diff scores in the two groups, but there was no significant difference between the SP score of the two groups. The results of a clinical trial performed by Ekvall Hansson et al. after performing vestibular-balance rehabilitation exercises in the intervention group showed a significant difference in reducing dizziness symptoms and improving quality of life, which were consistent with the results of this study ${ }^{13}$. SPNT and SP values in the intervention group were significantly increased, but no significant difference was observed in the control group. In other words, the results of this study indicate the effectiveness of rehabilitation exercises on the treatment of disability caused by dizziness and its components in patients with WAD. The results of showed the rehabilitation therapy caused a $25 \%$ improvement in neck reflex, extension and right side bending and a $50 \%$ improvement in motion for left side bending and left rotation, and treatment of symptoms in head movements with open and closed eyes, which were consistent with the results of this study ${ }^{16-24}$. Before the intervention, there was no significant difference between the mean scores of components and the total score of the DHI in the two groups $(p>0.05)$. After performing vestibular-balance rehabilitation exercises, there was a significant difference between the mean total score and the score of functional, physical, and emotional components in the two groups. These components were significantly improved in the intervention group but did not show any significant difference in the control group ${ }^{25-27}$. The results of a study by Tuo $\mathrm{K}$ et al. ${ }^{28}$ reported the treatment of dizziness, improving functional level and patient balance, and complete return to previous activities after 3 weeks of vestibular-balance rehabilitation exercises, which were consistent with the results of the present study ${ }^{11}$. The results of a study by Ekvall Hansson et al. ${ }^{4}$ showed a significant difference and improvement in the score of the physical and functional components of the DHI after 8 weeks and 3 months performing vestibular-balance rehabilitation exercises in the intervention group, which were consistent with the results of this study ${ }^{13}$. It showed that three weeks of rehabilitation based on open and closed eye exercises reduced pain, decreased dizziness, improved static balance, non-deviation in walking with both open and closed eyes, which was consistent with the results of the present study. The results of a metaanalysis study by Lystad et al. ${ }^{12}$. It reported the efficacy of rehabilitation therapy on treating dizziness, muscle tenderness, and pain, and improving range of motion in the neck, which was consistent with the results of the present study ${ }^{29}$.

\section{CONCLUSION}

Vestibular-balance rehabilitation exercises can be used as an effective treatment to treat dizziness and reduce the disability caused by it, followed by increasing the WAD patient's quality of life.

\section{CONFLICT OF INTEREST}

The Author declares no potential conflict of interest on publishing this paper

\section{ACKNOWLEDGEMENTS}

This study was funded by the Iran University of Medical Sciences (Code: 96-04-32-31835)."

\section{REFERENCES}

1. Robert C, kessinger DC and Dessay V Boeva DC. Vertigo, tinnitus, and hearing loss in the geriartic patient. J Manipulative Physiological Therap. 2000;23:352-362.

2. Rodriquez AA, barr KP, Burns SP. Whiplash: pathophysiology, diagnosis, treatment, and prognosis. Muscle Nerve. 2004;29:768 81.

3. Spitzer WO, Skovron ML, Salmi LR, Cassidy JD, Duranceau J, Suissa $\mathrm{S}$, et al. scientific monograph of the Quebec task force on whiplash associated disorders: redefining whiplash and it's management. Spine. 1995;20(8suppl):8-58.

4. Eva HE, Mansson NO, Ringsberg KA, Hakasson A. Dizziness among patients with whiplash-associated disorder: a randomized controlled trial. J Rehabil Med. 2006;38;387-390.

5. Treleaven J, Jull G, Lowchoy N. Smooth pursuit neck torsion test in whiplash-associated disorders: relationship to self-reports of neck pain and disability, dizziness and anxiety. J Rehabil Med. 2005;37:219-23.

6. Tjell C, Rosenhall U. smooth puruit neck torsion test: a specific test for cervical dizziness. Am J Otol. 1998;19:76-81.

7. Tuo KS, Cheng YY, Kao CL. Vestibular rehabilitation in a patient with whiplash-associated disorders. J Chin Med Assoc. 2006;69(12):591-5.

8. Lindsay W. sarbeck PT. Management of cervicogenic dizziness using vestibular rehabilitian and manual therapy: A case series on diagnosis and treatment.

9. Revel M, Minguet M, Gregoy P, Vailant J, Manuel JL. Changes in cervicocephalic kinesthesia after a proprioceptive rehabilitation program in patients with neck pain: a randomized controlled study. Arch Phys Med Rehabil. 1994;75:895-9.

10. Holm L, cassidy JD, sjogren $\mathrm{Y}$, nygren A. Impairment and work disability due to whiplash injury following traffic collisions. An analysis of insurance material from the Swedish road traffic injury commission. Scand J Public Health. 1999;27:116-123.

11. Sterner $Y$, Gerdle B. Acute and chronic whiplash disorders- a review. J Rehabil Med. 2004;36:193-209.

12. Wrisley DM, Sparto PJ, Whitney SL, Lystad K, Furman JM. Cervicogenic dizziness: A review of diagnosis and treatment. J Orthopedic Sports Phys Ther. 2000;30(12):755-66.

13. Dario C, Alpini N, whiplash injurie book. Diagnosis and Treatment. 2014

14. Petersen BW, Goldberg J, biolotto G, Fuller JH. cervicocollic reflex: its dynamic properties and interaction with vestibular reflex. J Neurophysiol. 1985;54;90-109.

15. Pyykko I, Schalen L, Wennmo C. single unit activity in flocculus of alert cat during vestibular, visual and proprioceptive stimulation. Agressologie. 1983;24;215-6.

16. Hirai $\mathrm{N}$, Hongo $\mathrm{T}$, sasaki $\mathrm{S}$. Neck muscle afferent input to spinocerebellar tract cells of the central cervical nucleus in the cat. Exp Brain Res. 1984;55;286-300. 
17. Hongo T, kitama T, Yoshida K. Integration of vestibular and neck afferent signal in the central cervical nucleus in pompeiano, allum Journal, edition. Vestibular control of posture and movement. Prog Brain Res. 1988:155-62.

18. Popova LB, Ragnarson B, Orlovsky GN, Grant G. Responses of neurons in the cenral cervical nucleus of the rat to proprioceptive and vestibular inputs. Archives Ital Boil. 1995;133:31-45.

19. Tjell C, Tenenbaum A, Sandstrom S. smooth pursuit neck torsion test- a specific test for whiplash associated disorder? J Whiplash and Related Disorders. 2003;1:9-24.

20. Barlow D, Freedam W. cervico-ocular reflex in the normal adults. Acta Otolaryngol. 1980;89;487-96.

21. Botros G. The tonic oculomotor function of the cervical joint and muscle receptor. Adv Otolaryngol. 1979;25;214-20.

22. Carlsson J, rosenhall U. oculomotor disturbances in patients with tension headache treated with acupuncture or physiotherapy. Cephalagia. 1990;10:123-9.

23. Treleaven J, Jull G, Sterling M. Dizziness and unsteadiness following whiplash injury: characteristic features and relationship with cervical joint position error: J Rehab Med. 2003;35:36-43.
24. Jacobson GP, Newman CW. The development of the dizziness handicap inventory. Arch Otolaryngol Head Neck Surg. 1990;116(4):424-7.

25. Poon DM, Chow LC, Au DK, Hui Y, Leung MC. Translation of the dizziness handicap inventory into Chinese, validation of it, and evaluation of the quality of life of patients with chronic dizziness. The annals of Otology Rhin Lary. 2004;113(12):1006-11.

26. Jacobson GSN. Balance function assessment and management: Plural Publishing. 2008.

27. Piker EG, Jacobson GP, Tran AT, Mccaslin DL, Hale ST. spouse perceptions of patients self-reported vertigo severity and dizziness. Oto Neuro. 2012;33(6):1034-9.

28. Tuo $\mathrm{K}$, cheng $\mathrm{Y}$, kao $\mathrm{CH}$. Vestibular rehabilitation in a patient with whiplash-associated disorders. J Chin Med Assoc. 2006;69(12):5915.

29. Oostendorp $A B$, vaneupen AJM, Vanerp JMM, Elvers HWH. Dizziness following whiplash injury: A neuro-otological study in manual therapy practice and therapeutic implication. J Manual Manipul Ther. 1999;7:123-130. 